\title{
Indicadores de sustentabilidad: utilidad y limitaciones
}

\author{
Reyna María Ibáñez Pérez* \\ Universidad Autónoma de Baja California Sur
}

\section{Resumen}

Desde su controversial origen, el modelo basado en el desarrollo sustentable (DS) ha generado numerosas críticas, algunas relacionadas con su carácter utópico, y otras, con su difícil seguimiento y medición. En respuesta a ello, se han propuesto indicadores de sustentabilidad (Is) como un instrumento útil que permita medir qué tan lejos estamos de ese esquema idealista de desarrollo. Hoy, las críticas no sólo se concentran en cuestionar el concepto y su posible aplicación, sino también en la validez y las limitaciones de esos indicadores. El debate se fundamenta en algunos de los siguientes argumentos: primero, algunos caen en el error de la conmensurabilidad; segundo, se requiere gran cantidad de información, así como técnicas costosas y sofisticadas, para calcularlos; tercero, su diseño impide implementarlos a pequeñas escalas, y cuarto, se ha desvirtuado su aplicación, al vincularlos con un mero discurso político. En este contexto, el objetivo del presente artículo es realizar un análisis crítico sobre las limitaciones de los is, con especial interés en las metodologías más empleadas tanto en el nivel nacional como en el mundial. Con base en opiniones críticas por parte del autor, revisión bibliográfica y entrevistas, se elaborará un análisis de fortalezas, oportunidades, debilidades y amenazas (FODA), a partir del cual se emitirán algunas recomendaciones.

\section{Palabras clave}

Desarrollo, indicadores, sustentabilidad, metodología, modelo, FODA.

*Correo electrónico: ribanez@uabcs.mx 


\title{
Sustainability indicators: their uses and limitations
}

Reyna María Ibáñez Pérez

Universidad Autónoma de Baja California Sur

\begin{abstract}
Since its controversial origin, the model based on sustainable development (DS), has generated much criticism, some related to its utopian nature and others related to monitoring and measurement difficult. In response, have been proposed sustainability indicators (IS) as a useful tool to measure, how far are we from the ideal scheme of development. Today, criticism focused not only on the concept and its possible application, but also the validity and limitations of these indicators. The discussion is based on some of the following arguments: first, some fall into the error of the commensurability, and second, it requires a lot of information, expensive and sophisticated techniques for calculating and thirdly, the design prevents its application to small scales and fourth, it has undermined its implementation, by binding to a mere political discourse. In this context, the aim of this paper is to critically analyze the limitations of the is, with particular interest in the methodologies employed mostly in the national and global scale. Based, critical opinions from the author, literature review and interviews, produce an analysis of strengths, weaknesses, opportunities and threats (SWOT) from which will be issued a series of recommendations.
\end{abstract}

\section{KEY WORDS}

Development, indicators, sustainability, methodology, model, swot. 


\section{Introducción}

En las últimas décadas se ha observado un importante deterioro en el ambiente, y a la par, la concentración de la pobreza se ha agudizado. Ante tal panorama, diversos organismos, gobiernos y la sociedad civil exigen un cambio que permita romper con estas tendencias desoladoras. Esto ha llevado a que desde finales de los sesenta se aglutinen presiones y elementos políticos, económicos y sociales, que al paso de los años recaerían en la propuesta de un nuevo modelo de desarrollo, el tan popular desarrollo sustentable (DS), para cuyo seguimiento se han elaborado una serie de indicadores y, con todo y sus controversias, se han realizado más de cien iniciativas para su aplicación; en muchos países son empleados para evaluar y reorientar sus políticas de desarrollo.

Así pues, en este artículo se ofrece un estudio y una reflexión sobre el estado actual y la aplicación de los indicadores de sustentabilidad (IS). Para tal efecto, en el apartado de antecedentes se hace un breve relato sobre el origen y vínculo de los indicadores con el tema de la sustentabilidad, además de mencionar algunos criterios usados para su clasificación, ponderación y categorización. Después, se describe la metodología empleada para elaborar este artículo, la cual se sustentó esencialmente en: análisis crítico de información, revisión bibliográfica, entrevistas a expertos, y metodología FODA (fortalezas, oportunidades, debilidades y amenazas). Se continúa con la revisión de las limitaciones y las ventajas de los indicadores de sustentabilidad, para lo cual se parte de un análisis general del tema; cabe mencionar que los resultados presentados se organizaron con base en el método antes referido. Enseguida, se lleva a cabo una discusión crítica relativa a las limitaciones de los modelos y enfoques más utilizados en el análisis del Ds: el enfoque presión, estado, respuesta (PER); fuerzas motrices-presión-estado-impactorespuesta (FPEIR), y el barómetro de la sustentabilidad. Para terminar, se comentan algunas reflexiones y recomendaciones en relación con los retos en materia del uso y aplicación de tales indicadores.

Independientemente del enfoque, cualquier modelo vinculado al DS debe descansar en una visión multidisciplinaria, que rompa con sus restricciones intrínsecas $\mathrm{y}$, sobre todo, considere las especificidades culturales de las zonas donde estos 104 modelos pretendan ser aplicados. 


\section{Antecedentes}

Los indicadores son herramientas para clarificar logros objetivos e impactos; también permiten detectar tendencias o ciertos fenómenos. Regularmente están diseñados para contar con estándares contra los cuales evaluar, estimar o demostrar el progreso de variables con respecto a metas establecidas. Hoy en día existen varios tipos y modelos de indicadores.

En particular, los Is intentan relacionar la información ambiental con la económica y la social, a fin de generar información ya sea sobre la contaminación, el deterioro del desarrollo productivo o el bienestar alcanzado por la población.

\section{El origen de los indicadores y su vínculo con el desarrollo sustentable}

Los Is tienen su origen en una larga historia de pensamiento sobre cómo medir el bienestar y sus diversas dimensiones (Max-Neef, 1991). Se vinculan al DS por ser un modelo que redefine el concepto de bienestar y que a su vez denota una fuerte preocupación por llegar a una situación tal que las generaciones presentes y futuras satisfagan sus necesidades por medio de un uso racional y equitativo de los recursos existentes. En el intento de medir qué tanto nos acercamos a esa situación surgen los IS, sugeridos con la implementación de la Agenda 21, en virtud de que facilitarían evaluar y reducir las brechas entre la realidad y las metas establecidas, en materia de sustentabilidad económica, social, ambiental e institucional. No obstante, su aplicación se oficializó al crear la Comisión de Desarrollo Sostenible (CDS), cuya tarea fue poner en marcha, mundial y nacionalmente, un sistema operativo (CDS, 1995) basado en un marco de evaluación que engloba criterios que van mucho más allá. Desde entonces se han documentado más de 129 iniciativas. Destaca América del Norte, que tiene el mayor número de propuestas, las cuales se vinculan especialmente con el tema del DS (Nieto, 2000-2001). 


\section{Características, tipologías y requisitos de un indicador}

En general, los Is poseen las siguientes características:

1. Se expresan como una señal física o numérica

2. Están inscritos en un marco teórico, asociado al Ds y al evento o problema que se pretende estudiar

3. Pueden variar sus unidades de medida

4. Regularmente, pueden ser expresados en escala de 0-1 o de 0-100\%

5. Generan información útil para realizar comparativos

6. Se enfocan en evaluar algún aspecto específico: economía, sociedad, ambiente [Mondragón Pérez, 2002]

Además, pueden ser susceptibles de clasificarse en función de diferentes criterios (Álvaro, 1994; Escudero, 2001; Dávila, 2004; Rotondo y Vela, 2004; Vila, Dávila Quintana y Mora, 2010; Ibáñez, 2010; Winograd, Eade Moldan y Billhartz, 1997):

1. Según la dimensión del DS al que están orientados. Cuando se hace referencia a las dimensiones del DS, se habla de sus alcances temáticos: sociales, económicos y ambientales. Aunque con el paso de los años se han propuesto nuevas dimensiones y áreas temáticas, por lo regular se tiende a clasificar los is en económicos (ejemplo: ingreso per cápita), sociales (ejemplo: analfabetismo), ambientales (ejemplo: territorio protegido), y de otra índole o tema específico (ejemplo: tenencia de la tierra).

2. Según el sentido teórico y la obtención de datos para su elaboración. Se puede distinguir entre indicadores objetivos y subjetivos:

a) Indicadores objetivos (duros). Se fundamentan en evidencias externas independientes del informante (ejemplo: calidad del agua, emisión de partículas contaminantes), suponiendo que los métodos de captación y procesamiento de la información son objetivos.

b) Indicadores subjetivos (alternos). Reflejan percepciones y opiniones de la población respecto a su situación (ejemplo: percepción de la participación social). 
3. Según el modelo en el que estén basados. Los modelos más usados en el ámbito internacional son los basados en el enfoque PER, el enfoque FPEIR $\mathrm{y}$, en menor medida, los que se asocian al barómetro de la sustentabilidad.

a) Basados en el modelo PER. Modelo desarrollado por la Organización para la Cooperación y el Desarrollo Económicos (OCDE, 2003) con la finalidad de contar con información que permitirá estructurar su trabajo sobre políticas ambientales. Parte del supuesto de que las actividades humanas ejercen una presión sobre el medio ambiente, y que éste, a su vez, registra cambios de estado en función de ellas, y la sociedad responde con la adopción de medidas que tratarían de mantener los equilibrios ecoló-gicos que le parecen adecuados. Representa estas relaciones de causa-efecto por medio de indicadores, mostrando cómo están interrelacionados el ambiente, la economía y la sociedad. Los indicadores propuestos en este modelo son:

- Indicadores de presión. Describen la presión ejercida por las actividades humanas sobre el medio ambiente y los recursos naturales; están relacionados con los patrones de producción y de consumo. Un caso de presión sobre la calidad del aire son las emisiones de gases contaminantes a la atmósfera.

- Indicadores de estado. Diseñados para mostrar imágenes (estado) de la situación que guardan el medio ambiente y los recursos naturales en el tiempo. Por ejemplo, la superficie cubierta por bosques.

- Indicadores de respuesta. Vinculados con acciones y reacciones colectivas que lleva a cabo la sociedad para mitigar, adaptar y revertir los efectos negativos de las actividades humanas sobre el medio ambiente. Ejemplo de ello es el porcentaje de territorio dedicado a conservación.

b) Basados en el modelo FPEIR. Modelo sustentado en la idea de que las actividades humanas (fuerzas motrices) ejercen presión sobre el medio físico y, en consecuencia, su estado cambia, lo cual impacta la salud humana, los ecosistemas y los recursos. Esta situación da lugar a respuestas de las sociedades humanas, incidiendo en las fuerzas motrices, en las presiones, o en el estado o los impactos directamente (Cifrián et al., 
2006). Puesto que este modelo se fundamenta en el esquema per, utiliza indicadores de presión, estado y repuesta, y su aportación como tal consiste en tratar de perfeccionar aquel esquema, para lo cual propone un nuevo tipo de indicadores: de impacto, que son usados para describir cambios en las condiciones del medio, como la tasa de deforestación de los bosques o el número de especies extintas.

c) Según el modelo para el análisis de la sustentabilidad, basado en el barómetro de la sustentabilidad (Unión Internacional para la Conservación de la Naturaleza-UICN, 2001). Este modelo parte de que el ds puede medirse si se determina cuál es el nivel de bienestar del ambiente y de las personas. Para tal efecto, propone 40 indicadores (principalmente de presión, impacto y respuesta), agrupados en dos componentes (social y ecológico), cuyos resultados deben ser ubicados en los ejes del barómetro de la sustentabilidad (véase figura 1). Esto permite ilustrar y categorizar el grado de sustentabilidad de la zona que se estudia, y sobre todo ayuda a determinar hacia cuál componente se deben enfocar mayores esfuerzos.

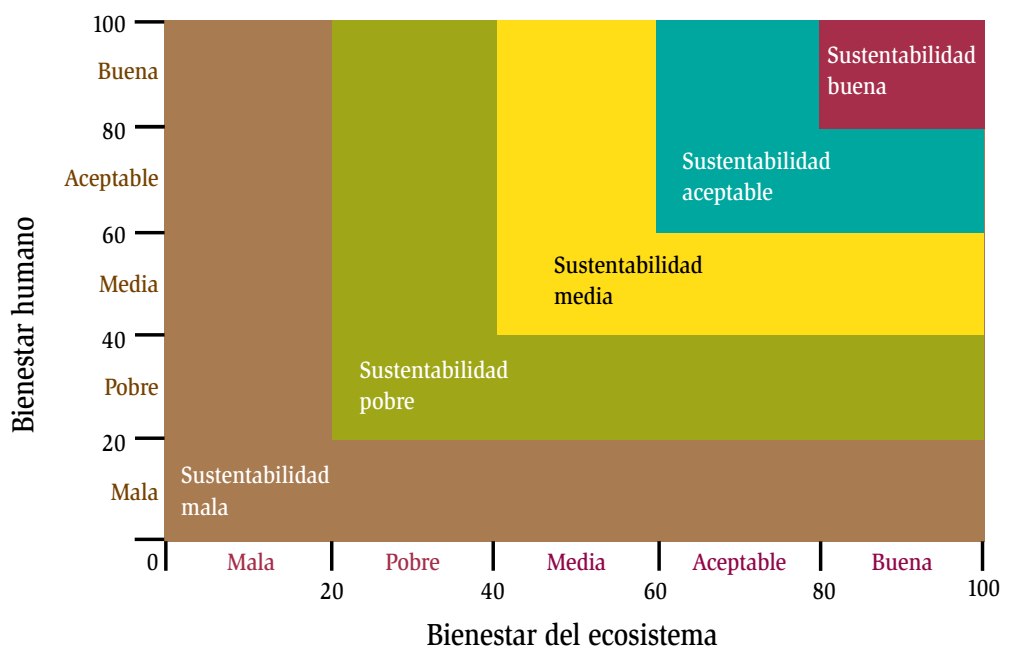

Fuente: UICN, 2001. 
1. Según el enfoque en el que estén basados. En este caso hay dos clasificaciones:

a) Monetarios. Intentan establecer valorizaciones monetarias sobre la amortización de los recursos y servicios ambientales y su impacto en el nivel de vida de la población. Por ejemplo, el PIB verde, el índice de bienestar económico sustentable (IBES) y las cuentas patrimoniales (véase cuadro 1). La mayoría de los indicadores monetarios tiene alcance mundial y nacional. Por su naturaleza, al igual que otros modelos y enfoques, resulta difícil implementarlos a escalas territoriales pequeñas.

\section{CUADRO 1. Indicadores monetarios relacionados con el DS, alcances y objetivos}

Autor

Alcance

Xiaohua, Mundial y Intenta aportar criterios para llegar a valo2007 nacional rizaciones moneta-rias consensuadas, tanto de la amortización de los recursos naturales como de los servicios ambientales

Índice de

bienestar

económico

sustentable

(IBES)

Cuentas

patrimoniales

(monetizadas)
Daly y Cobb, Mundial 1989

Sejenovich, Mundial y Busca analizar la reproducción de la natu1996

Considera una amplia variedad de factores sociales y ambientales, como la distribución del ingreso, la contribución de las actividades no monetarizadas, el agotamiento de los recursos naturales y los daños ecológicos, medidos a través de la acumulación de gastos defensivos nacional taria del balance de la captación y consumo de materiales, energía, agua, entre otros

Fuente: Elaboración propia con base en Achkar et al., 2005.

b) Biofísicos. Miden físicamente los insumos usados en los distintos productos y servicios de la economía en relación con su vida útil, niveles de consumo e impacto ambiental. Por mencionar algunos: mochila ecológica (MIPS), huella ecológica (HE), espacio ambiental (EA), capacidad de carga (CC), índice ambiental sustentable o de sustentabilidad ambiental (ISA) e índice del planeta viviente (IPV) (véase cuadro 2). Este tipo de indicadores intenta medir flujos disponibles o consumidos de bienes y servicios ambientales, para lo cual se requiere información obtenida de manera objetiva, con alto rigor científico y metodológico. 
CUADRO 2. Indicadores biofísicos relacionados con el DS, alcances y objetivos

\begin{tabular}{|c|c|c|c|c|}
\hline \multirow{7}{*}{ 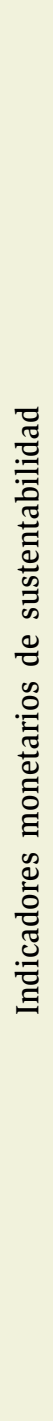 } & Indicador o índice & Autor & Alcance & Objetivo \\
\hline & $\begin{array}{l}\text { Mochila ecológica } \\
\text { (MIPS) }\end{array}$ & $\begin{array}{l}\text { Schmidt-Bleek, } \\
\text { 1994; Spangen- } \\
\text { berg, y Schmidt- } \\
\text { Bleek, } 1997\end{array}$ & Mundial & $\begin{array}{l}\text { Mide físicamente en toneladas los } \\
\text { insumos (inputs) usados en los } \\
\text { distintos productos y servicios de } \\
\text { la economía en relación con su } \\
\text { vida útil; intenta evaluar si existe } \\
\text { una desmaterialización de la eco- } \\
\text { nomía y realizar un seguimiento } \\
\text { en el tiempo }\end{array}$ \\
\hline & Huella ecológica & $\begin{array}{l}\text { Wackernagel } \\
\text { y Rees, } 1996\end{array}$ & $\begin{array}{l}\text { Mundial } \\
\text { y } \\
\text { nacional }\end{array}$ & $\begin{array}{l}\text { Mide el impacto de una persona, } \\
\text { ciudad o país, sobre la tierra, al } \\
\text { satisfacer lo que consume y para } \\
\text { absorber sus residuos }\end{array}$ \\
\hline & Espacio ambiental & $\begin{array}{l}\text { Spangenberg, } \\
1999\end{array}$ & $\begin{array}{l}\text { Mundial } \\
\text { (conceptual) }\end{array}$ & $\begin{array}{l}\text { Intenta medir la cantidad de re- } \\
\text { cursos naturales renovables y no } \\
\text { renovables que podemos usar (y } \\
\text { los niveles de desperdicios y con- } \\
\text { taminación que podemos permitir- } \\
\text { nos) sin privar a las generaciones } \\
\text { futuras de su derecho al mismo } \\
\text { uso de los recursos naturales }\end{array}$ \\
\hline & Capacidad de carga & $\begin{array}{l}\text { Wackernagel } \\
\text { y Rees, } 1996\end{array}$ & $\begin{array}{l}\text { Casos } \\
\text { específicos } \\
\text { e intereses } \\
\text { académicos }\end{array}$ & $\begin{array}{l}\text { Determina el nivel de población } \\
\text { que puede soportar un medio am- } \\
\text { biente dado sin sufrir un impacto } \\
\text { negativo significativo (número } \\
\text { máximo de individuos que puede } \\
\text { soportar una superficie) }\end{array}$ \\
\hline & $\begin{array}{l}\text { Índice de } \\
\text { sustentabilidad } \\
\text { ambiental (ISA) }\end{array}$ & YCELP/CIESIN, 2005 & $\begin{array}{l}\text { Mundial } \\
\text { (conceptual) }\end{array}$ & $\begin{array}{l}\text { Busca establecer en qué medida } \\
\text { se realiza una gestión de los im- } \\
\text { pactos negativos sobre el medio } \\
\text { ambiente }\end{array}$ \\
\hline & $\begin{array}{l}\text { Índice del planeta } \\
\text { viviente (IPV) }\end{array}$ & UNEP-WCMC, 2000 & Mundial & $\begin{array}{l}\text { Pretende evaluar el estado de la } \\
\text { biodiversidad mundial a partir } \\
\text { de la medición de las tendencias } \\
\text { en las poblaciones de diferentes } \\
\text { especies de vertebrados que habi- } \\
\text { tan ambientes terrestres, marinos } \\
\text { y dulceacuícolas }\end{array}$ \\
\hline
\end{tabular}




\section{Criterios más utilizados en la categorización y ponderación de IS}

Los indicadores pueden ponderarse y categorizarse con base en propuestas de expertos (método Delphi), en opiniones recabadas en foros de participación, o simplemente tomando criterios establecidos en la literatura (véase cuadro 3).

CUADRO 3. Criterios más utilizados en la categorización de IS

\begin{tabular}{lllc}
$\begin{array}{c}\text { Índice de } \\
\text { desarrollo humano } \\
\text { (IDH) PNUD, 2008 }\end{array}$ & Prescott-Allen, 1997 & $\begin{array}{c}\text { Barómetro de } \\
\text { sustentabilidad } \\
\text { UICN, 2001 }\end{array}$ & Rango (resultado) \\
Alto & Excelente & Ideal (deseable) & $\mathbf{0 . 8 - 1 . 0}$ \\
Medio & Bueno & Bueno & $\mathbf{0 . 6 - 0 . 8}$ \\
& & & \\
& Medio & Medio & $\mathbf{0 . 4 - 0 . 6}$ \\
Bajo & Malo & Pobre & $\mathbf{0 . 2 - 0 . 4}$ \\
& Muy malo & Malo (indeseable) & $\mathbf{0 . 0 - 0 . 2}$ \\
\hline
\end{tabular}

Fuente: Ibáñez, 2010; Ivanova et al., 2010.

Estos criterios pueden ser empleados en diferentes escalas para determinar los valores individuales de cada indicador, de manera que se puede optar por expresiones en porcentajes o en escala del 0 al 1, lo cual facilitará tanto su manejo como su interpretación. Un indicador que presente un valor de 1 implicará un impacto absolutamente positivo en el bienestar; por ejemplo, si se obtiene un valor de 1 en servicios educativos, significa que hay una cobertura de $100 \%$ en ese tipo de servicios.

Por otro lado, cuando es posible reunir información de un conjunto de indicadores (económicos, sociales y ambientales), es factible resumirla en un promedio o índice, el cual puede estar diseñado para expresar el grado de sustentabilidad (medida que señala qué tanto nos acercamos al DS). Por ejemplo, según el método de la UICN (2001), obtener un resultado promedio entre 0.8 y 1.0 expresa un nivel deseable. No obstante, un resultado de 0.0-0.2 denota una situación totalmente indeseable, así como un fuerte rezago social, combinado con una pésima calidad ambiental. 
Es importante aclarar que independientemente del tipo de indicador y el enfoque usado en su elaboración, es indispensable que, para garantizar su validez, éste cumpla con algunos requisitos: precisión, consistencia, facilidad de recolección, disponibilidad, relevancia, pertinencia y confiabilidad (OCDE, 1998).

\section{Metodología}

Para realizar este trabajo se utilizaron las metodologías que se explican a continuación.

\section{Revisión bibliográfica y documental}

Se consultaron estudios de organizaciones internacionales y nacionales pioneras en el desarrollo de Is, como las siguientes:

1. Propuestas de organismos multinacionales, en particular de la Comisión Económica para América Latina y el Caribe (CEPAL) (Quiroga Martínez, 2007), la OCDE (1998 y 2003), la CDS (1995 y 1996), el Programa de las Naciones Unidas para el Desarrollo (PNUD, 2008), la Agencia de Protección Ambiental (EPA, 1994), la Organización Mundial de Turismo (OMT, 1997), la UICN (2001), el Fondo Mundial para la Naturaleza (WWF, 2010) y la Asociación de Estados del Caribe (AEC, 1999).

2. Propuestas nacionales y regionales, como la de la Secretaría de Medio Ambiente y Recursos Naturales (Semarnat, 2010), el Instituto Nacional de Ecología y el Instituto Nacional de Estadística, Geografía e Informática (INE/INEGI, 2000), la Comisión Nacional de Áreas Naturales Protegidas (Conanp, 2007) y la Secretaría de Turismo (Sectur, 2008).

3. Investigaciones realizadas por expertos, tales como Cornelissen et al. (2001), Daly y Cobb (1989), Quiroga Martínez (2001), Nieto (2000-2001) y Wackernagel y Rees (1996).

4. Análisis de material complementario, como artículos y trabajos de tesis vinculados con la temática, en particular Ibáñez (2010) y Sánchez (2010). 


\section{Entrevistas con expertos}

Éstas se dirigieron a cinco especialistas locales y se llevaron a cabo en 2010.

\section{Análisis FODA}

Esta herramienta tiene múltiples aplicaciones, puesto que permite conformar un cuadro de la situación actual de un tema o controversia, de una región, sector 0 empresa. Además, facilita obtener un diagnóstico para la toma de decisiones, acordes con objetivos formulados respecto al tema y ámbito de estudio (Ponce Talancón, 2006). Cabe señalar que la elaboración del FODA se basó en un análisis crítico y en opiniones propias sustentadas en las fuentes de información descritas en el apartado anterior. Los resultados obtenidos se analizaron considerando la utilidad y limitaciones de los Is. Posteriormente, se realizó una clasificación de sus fortalezas (aspectos internos que favorecen su desarrollo), oportunidades (condiciones disponibles para mejorar), debilidades (factores intrínsecos que pueden anular las fortalezas) y amenazas (factores externos que restringen su adecuado desarrollo).

\section{Resultados}

La revisión de los documentos y fuentes antes mencionados, y en especial de los textos de Fürst (2010), Wautiez y Reyes (2007), Sánchez (2010) y Martínez Alier (1999), contribuyó a delinear las críticas que se presentan en este apartado, cuyos resultados indican que, independientemente del enfoque empleado, existen las fortalezas, debilidades, oportunidades y amenazas expuestas en el cuadro 4. 
CUAdRO 4. Análisis foda de las limitaciones y ventajas de los IS

\section{FORTALEZAS}

- Ofrecen una visión de las condiciones ambientales y respuestas de una sociedad o gobierno

- Son sencillos de interpretar, siempre que se expresen en escala de 0 a 1,0 de 1 a $100 \%$

- En algunos casos permiten categorizar el nivel de sustentabilidad

- Proporcionan información sobre el estado de una o varias de las dimensiones del desarrollo sustentable

- Generan un diagnóstico del grado de avance hacia el desarrollo sustentable

- Crean información útil para reorientar políticas de desarrollo

- Facilitan la difusión de información a diferentes grupos de usuarios y a la sociedad en su conjunto

- Permiten establecer comparativos, según la escala en que hayan sido aplicados

\section{OPORTUNIDADES}

- Compromisos internacionales que fomentan su implementación

- La mayoría de los países del orbe le ha otorgado validez y obligación legal

- Organismos internacionales acrecentan recursos para su medición

- Tendencia generalizada a buscar prácticas, procesos y métodos relacionados con el uso responsable del ambiente

\section{DEBILIDADES}

- En algunos casos se requiere información compleja y detallada

- Para establecer tendencias se ocupan datos agrupados en series de tiempo

- Su estimación puede ser muy costosa y a veces requiere técnicas complejas

- No se ha logrado un consenso sobre la categorización y ponderación de sus efectos en el bienestar

- No existen criterios sobre la cantidad mínima de indicadores en un modelo de sustentabilidad

- Algunos de los indicadores biofísicos recaen en la inconmensurabilidad

- No siempre pueden ser utilizados en diferentes escalas

- Se requiere una visión multidisciplinar para su aplicación y manejo

- Al usar indicadores subjetivos, se incurre en el riesgo de obtener información no confiable

\section{AMENAZAS}

- La sustentabilidad se ha ido incorporando a un mero discurso político

- En países de Latinoamérica, los indicadores se usan más como un medio para cumplir compromisos internacionales que para implementar medidas con las cuales elevar la calidad ambiental

- En algunos países, su aplicación en temáticas específicas (manejo de áreas naturales) se enfoca a medir metas burocráticas, más que el mismo avance en el tema de sustentabilidad 
CUADRo 4. Análisis foda de las limitaciones y ventajas de los IS

(Finaliza)

OPORTUNIDADES

- La preocupación socioambiental se ha generalizado y se busca determinar el grado de deterioro del medio ambiente y el nivel de rezago en la sociedad

- Se reconoce mundialmente su utilidad para evaluar y dar seguimiento a políticas de desarrollo
AMENAZAS

- Poco a poco se ha desvirtuado su razón de ser y se pone en entredicho su validez y utilidad

- La complejidad de las dimensiones del desarrollo sustentable hacen difícil la creación de indicadores confiables, representativos y razonables en términos de costo- beneficio

Fuente: Elaboración propia.

Como se puede observar, las críticas y debilidades se fundamentan principalmente en los siguientes aspectos:

1. El problema de las escalas (entendido en términos del tamaño del espacio o área geográfica) parece ser un obstáculo para medir el grado o nivel de sustentabilidad en países o localidades pequeñas en vías de desarrollo, donde la atención gubernamental y la planeación del desarrollo se concentran en ámbitos o problemas relacionados con aspectos macroeconómicos

2. La complejidad y altos costos en algunas de sus metodologías, puesto que las mediciones basadas en indicadores objetivos regularmente se asocian a altos costos y complejas técnicas para la obtención y procesamiento de los datos; como consecuencia, los países que carecen de tal infraestructura prolongan la implementación de indicadores

3. La escasa información y datos para su medición actual o futura obliga a que los países que no cuentan con recursos suficientes implementen indicadores cualitativos o subjetivos, situación que influye negativamente en la confiabilidad de los datos generados a partir de tales métodos

4. La politización del tema de la sustentabilidad, es decir, la incorporación del tema del ds en un discurso político carente de conciencia y usado como un término aparentemente sofisticado

5. La inexistencia de reglas, técnicas o consenso general; para su calibración, categorización y ponderación, puede contribuir a su mala interpretación, fácil manipulación y escasa confiabilidad 
Pese a los puntos mencionados, los Is presentan fortalezas y oportunidades; por señalar algunas:

1. Bajo ciertos requisitos, como claridad, precisión, consistencia y confiabilidad, pueden ser un valioso instrumento para medir el estado de cada componente del DS

2. Proporcionan información útil en términos de políticas; esto favorecería la inducción de cambios tanto en el corto como en el largo plazo, mediante la aplicación de medidas para mejorar el bienestar económico, social o ambiental

3. Existe una tendencia generalizada a buscar prácticas, procesos y métodos relacionados con el uso responsable del ambiente; no obstante, se requiere un esfuerzo colectivo para pasar del discurso, el debate y la concepción de modelos teóricos (en algunas ocasiones inaplicables en la realidad) a acciones y compromisos verdaderos, que se traduzcan en la puesta en práctica de este mecanismo de medición tan provechoso

4. La mayoría de los países del mundo les han otorgado validez y obligación legal, por lo cual es menester superar las inercias que impiden su correcta aplicación (costos, carencia de datos, dificultades tecnológicas, desconocimiento, entre otras)

5. En concreto, a pesar de sus debilidades y amenazas, los Is pueden ayudarnos a acumular información para delinear estrategias encaminadas a lograr (en la medida de lo posible) una situación tal que las generaciones presentes y futuras puedan satisfacer sus necesidades, mediante el uso responsable de los recursos existentes y bajo condiciones de justicia y equidad. No obstante, se requiere mucho más que mera simulación.

\section{Discusión}

Las disyuntivas sobre los indicadores de sustentabilidad se agudizan al analizar el enfoque o tipo de indicador, por ejemplo en el caso de:

1161 . Indicadores monetarios de sustentabilidad. Las principales críticas giran en torno a la imposibilidad de asignar valor a daños al sistema ambiental cuando 
en muchas ocasiones son ya irreversibles o acumulativos. Además, al no conocerse con certeza la situación de las reservas de recursos y la capacidad de futuras tecnologías, resulta carente de toda ética intentar reducir la diversidad a una unidad común con valorizaciones monetarias (regularmente arbitrarias, dada la escasa información) actuales o futuras (Lutz, 1993; Bossel, 1996).

2. Indicadores biofísicos. El debate se concentra en que estos indicadores no permiten comparar situaciones fácilmente, y en que, por su naturaleza, muchas veces no son de aplicación universal. Pero las críticas más severas versan sobre dos aspectos: primero, que su principal objetivo es didáctico y no de investigación; segundo, que la información para sus cálculos regularmente no está disponible y por tanto son irrepetibles. Entonces ¿carecerían de validez?

3. Indicadores basados en el modelo PER. Si bien presentan inventarios muy útiles de información amplia y diferenciada sobre variables e indicadores en sus distintos niveles y categorías, esto se logra por medio de un costoso monitoreo científico que muchas veces implica el manejo de escalas específicas, regularmente nacionales y regionales; otra desventaja es que, por sí solos, no proporcionan datos precisos respecto al grado o nivel de sustentabilidad global, y en diversas ocasiones sus resultados no son sencillos de interpretar.

4. Indicadores basados en el modelo FPEIR. Dado que este modelo abarca distintos aspectos y dimensiones del desarrollo humano sostenible, por lo que es calificado como un enfoque más holista, se vuelve menos operable en la práctica, en virtud de la heterogénea y enorme cantidad de datos requeridos.

5. Indicadores y enfoque basados en el barómetro de la sustentabilidad de la UICN. Aunque este enfoque parte del principio de equidad y de igual trato a personas y ecosistemas, una de sus principales deficiencias es que asume que existe información perfecta sobre cada una de las dimensiones que estudia. No obstante, en muchos casos (en particular para pequeñas localidades) falta información, y es imposible incorporar el mismo número de indicadores a cada dimensión. Además, al utilizar algunos indicadores del modelo PER, principalmente en la cuestión ecológica, se recae en problemas propios de ese modelo. 
Cabe señalar que si bien los modelos PER y FPEIR son los más empleados en iniciativas nacionales, ambos requieren indicadores duros, lo que los hace inoperables en países, regiones o localidades que no cuentan con los recursos necesarios para su estimación directa. Aunado a lo anterior, tienden a sugerir una relación causa-efectorespuesta que realmente no puede comprobarse como cierta; en su mayoría están orientados hacia indicadores referentes al output (emisiones, residuos, erosión) y estado de calidad, para cuyo registro y medición muchas veces no existen conocimientos certeros sobre el alcance y los riesgos de alteración ecosistémica; siguen sobre todo el principio de mitigación en lugar del de prevención; resulta difícil monitorear su desempeño, dada la desagregación de sus dimensiones, lo cual obstaculiza fijar metas (Fürst, 2010). Además, aunque requieren series consistentes de datos, no necesariamente es posible establecer relaciones lógicas entre los distintos indicadores. Asimismo, el enfoque con que estas metodologías están diseñadas sólo permitirá establecer el diagnóstico de la situación, pero no puede ser usado en programas o políticas con fines de prevención (Wautiez y Reyes, 2007). Con ello, la información resultante sólo hará posible formular políticas de corto plazo que no promueven cambios estructurales (Fürst, 2010).

Por su naturaleza metodológica, el modelo propuesto por la UICN es de difícil aplicación, en especial si se trata de diagnosticar la contribución de sectores o actividades específicas, por ejemplo el turismo, ya que no contempla el uso de indicadores propios de esta actividad, aunque quizá la solución sería complementarla con otras metodologías (como el caso de la OMT). Pero esa tarea puede devenir más compleja de lo que se llega a imaginar, ya que, de inicio, habría la necesidad de incorporar indicadores con otras características: tal vez menos objetivos, menos duros, y basados en métodos (encuestas, entrevistas, foros, etc.) no aceptados por completo en algunas disciplinas.

Así pues, los intentos de integración de los enfoques antes mencionados están lejos de ser aceptados como esquemas coherentes, lo cual evidencia uno de los retos más importantes para la agenda de trabajo en materia de Is: cumplir con los requisitos que todo indicador exige (Fürst, 2010). En consecuencia, los sistemas de Is hasta hoy desarrollados siguen siendo cuestionados con severidad, en virtud de que aparentemente atienden necesidades científicas o académicas, lo que ocasio118 na que muchas veces éstos sean inoperables. 


\section{Conclusiones}

Es preciso señalar que, no obstante la importancia que han adquirido los IS, la mayoría de las metodologías en este ámbito se han ideado para medir el funcionamiento global o regional, lo que dificulta su implementación en escalas geográficas locales, y además tienden a no considerar especificidades culturales. Por tanto, son incapaces de ofrecer resultados ricos y prácticos. Asimismo, la falta de consensos en relación con la ponderación, categorización y tipo de indicadores a integrar en los modelos para el análisis de sustentabilidad genera confusión y obliga a que cada investigador o institución recurra a los métodos que considere pertinentes, ya sea basados en la literatura, en estándares internacionales, en foros participativos, en entrevistas o trabajo con expertos. Todo ello crea mayor polémica y pone en duda la pertinencia, la coherencia y la validez de los modelos propuestos.

Por otro lado, aunque se sabe que los indicadores deben cumplir ciertos requisitos esenciales, en la práctica es raro que todas esas cualidades se encuentren en un único indicador, ya que en muchas ocasiones éste puede resultar simple y fácil de entender e interpretar, aunque no sea científicamente válido (Curiel, 2001). También, de acuerdo con la disponibilidad de recursos y los objetivos que se busquen alcanzar con la formulación de indicadores para la sustentabilidad, existen diversos marcos o estructuras de análisis para la organización de un sistema o modelo de indicadores, cuya construcción puede estar sujeta a restricciones de información, de recursos económicos y humanos, y, desde luego, al eterno problema de las escalas, es decir, el ámbito espacial de aplicación.

En este contexto, es necesario crear modelos de sustentabilidad que incluyan nuevos criterios espaciales, culturales, sociales y políticos. Con la integración de indicadores más acordes a la realidad de un país o localidad, se obtendría información más fehaciente sobre el estado de las tres dimensiones del Ds, lo que permitiría fundamentar mejores medidas en materia de políticas encaminadas a mejorar la calidad de vida desde el punto de vista de la sustentabilidad.

\section{Recomendaciones}

Con base en el análisis de resultados y en la discusión, se han emitido las siguientes recomendaciones: 
1. Mayores esfuerzos en la recopilación de datos. Los esfuerzos gubernamentales deberían estar encaminados a establecer sistemas de estadísticas y monitoreo de los Is, sobre todo en áreas que han sido vinculadas con algún régimen de protección. De tal suerte que a mediano plazo fuese posible contar con series de tiempo de indicadores no medidos en la actualidad, lo cual podría contribuir al mejoramiento y mayor confiabilidad de los datos. Invariablemente, una sólida infraestructura estadística es cardinal, lo que requiere desarrollar, a lo largo del tiempo, la capacidad técnica y profesional para mantener y desplegar sistemas de acuerdo con estándares internacionales (Herrera Ulloa, 2004). Esto permitirá elaborar modelos más consistentes y rigurosos, en términos estadísticos, para el análisis de la sustentabilidad, de manera que los indicadores que los integren sean realmente significativos y no se incurra en problemas de redundancia, lo cual ayudará a construir tendencias o escenarios futuros que hagan factible analizar posibles relaciones entre los indicadores, sin importar su tipo.

2. Consideración de por lo menos un indicador de cada dimensión de la sustentabilidad. Aunque no existe una regla al respecto, es importante que se considere por lo menos un indicador de cada dimensión del DS, de lo contrario se incurre en omisión y reduccionismo.

3. Consideración de indicadores duros. Esto permitirá tener una idea clara y objetiva de los cambios en los medios social y natural (tasa de deforestación, especies en peligro de extinción, alfabetización, ingreso, delincuencia).

4. Integración de indicadores alternos. En casos en los que se carece de datos duros es posible estimar de manera indirecta algunas características que influyen en el nivel de sustentabilidad (programas, certificaciones ambientales).

5. Ponderación igualitaria de las dimensiones o componentes del modelo propuesto. Si bien no hay un consenso universal sobre el peso que se debe asignar a cada dimensión o indicador, se sabe que el concepto de Ds tiene implícito el principio de equidad (en todo sentido), con lo que la ponderación igualitaria de los componentes de un modelo atendería los umbrales y fundamentos teóricos de tal concepto.

6. Construcción de escenarios tentativos. La manipulación de indicadores críticos permitiría evaluar posibles cambios en la sustentabilidad. La construcción 
de estos escenarios puede partir de ciertas hipótesis o situaciones deseables 0 indeseables, amén de servir como ejercicio para comprender el impacto que las medidas de política podrían llegar a tener en el bienestar social, económico y ambiental.

7. Integración de nuevas tecnologías de la información. Como las técnicas de mapeo, que ofrecen un panorama más claro del estado de los medios social y natural. Sin embargo, se debe considerar que no todos los software están diseñados para manejar todas las escalas geográficas; por ejemplo, en los niveles regional, estatal y municipal se recomienda usar sistemas de información geográfica (SIG), mientras que para otras escalas, como en localidades pequeñas, es preferible un software menos especializado pero que, para efectos prácticos, permita obtener un panorama similar.

8. En la elaboración de propuestas metodológicas debe tenerse en cuenta lo siguiente:

a) Atender las especificidades de la zona de estudio

b) Incorporar indicadores propuestos que cumplan con algunos requisitos mínimos

c) Independientemente de la escala territorial, las metodologías planteadas deben incorporar cada una de las dimensiones del desarrollo sustentable, diseñarse con fines de diagnóstico y en cuanto instrumento para obtener información confiable, y hacer posible la toma de decisiones para mejorar el nivel de sustentabilidad de la zona en cuestión

Atender las recomendaciones anteriores implicará un trabajo multidisciplinario que permita replantear, adaptar o mejorar los enfoques existentes, en función de las fortalezas, oportunidades, debilidades y amenazas teóricas y prácticas de cada enfoque e indicador en particular, lo que representa el reto actual en la agenda de investigación sobre IS. 


\section{FUENTES CONSULTADAS}

Achkar, M. (2005). "Indicadores de sustentabilidad", en M. Achkar et al. Ordenamiento ambiental del territorio. Montevideo: Comisión Sectorial de Educación Permanente, Facultad de Ciencias, Univer-sidad de la República Uruguay, 55-70.

Achkar, M., et al. (2005). Ordenamiento ambiental del territorio. Montevideo: Comisión Sectorial de Educación Permanente, Facultad de Ciencias, Universidad de la República Uruguay.

AEC (1999). Declaración para el Establecimiento de la Zona de Turismo Sustentable del Caribe. II Cumbre de Jefes de Estado y/o Gobierno de la Asociación de Estados del Caribe, República Dominicana.

Álvaro, M. (coord.) (1994). Propuesta de un sistema de indicadores sociales de igualdad entre géneros. Madrid: Instituto de la Mujer.

Bossel, H. (1996). “Deriving Indicators of Sustainable Development”. Environmental Modeling and Assessment, 1, 193-218.

CDS (1995). Work Program on Indicators of Sustainable Development of the Commission of Sustainable Development. Nueva York: Comisión de Desarrollo Sostenible-Organización de las Naciones Unidas (ONU).

- (1996). Indicators of Sustainable Development Framework and Methodologies. Nueva York: Comisión de Desarrollo Sostenible-ONU.

Cifrián, E., et al. (2006). Indicadores ambientales y sistema de indicadores. Cuaderno 1. Cantabria: Consejería del Medio Ambiente/Punto Focal de Residuos de Cantabria.

Conanp (2007). Programa de Turismo en anp 2006-2012. México: Comisión Nacional de Áreas Naturales Protegidas.

Cornelissen, A. M. G., et al. (2001). "Assessment of the Contribution of Sustainability Indicators to Sustainable Development: a Novel Approach Using Fuzzy Set Theory”. Agriculture, Ecosystems and Environment, 86, 173-185.

Curiel, E. (2001). Análisis de indicadores de desarrollo de la educación ambiental en España. Tesis doctoral. Madrid: Universidad Complu-tense de Madrid.

Daly, H. E. y J. Cobb (1989). For the Common Good. Boston: Beacon Press.

122 Dávila, M. (2004). Indicadores de género. Guía práctica. Sevilla: Instituto Andaluz de la Mujer. 
EPA (1994). Indicators Development Strategy, Environmental Monitoring Assessment Program. Carolina del Norte: Programa de Monitoreo y Evaluación Ambientales, Agencia de Protección Ambiental.

Escudero, T. (2001). "Indicadores para el sistema universitario español”. Anuario de Pedagogía, 3, 131-142. Zaragoza: Universidad de Zaragoza.

Fürst, E. (2010). El debate actual sobre indicadores de sostenibilidad [en línea]. Costa Rica: Centro Internacional en Política Económi-ca, Universidad Nacional Heredia. Disponible en: http://sembrandofuturosostenibles.blogspot. mx/2010/01/el-debate-sobre-indicadores-de.html [2012, 27 de marzo].

Herrera Ulloa, Á. (2004). Indicadores de sustentabilidad en el desarrollo de la industria turística en Baja California Sur. Tesis doctoral. La Paz: Centro de Investigaciones Biológicas del Noroeste.

Ibáñez, R. (2010). Modelo para el análisis de sustentabilidad turística en pequeñas comunidades costeras, el caso de Cabo Pulmo, bcs. Avance de tesis para obtener el grado de doctora en Ciencias. La Paz: Universidad Autónoma de Baja California Sur (UABCS).

INE/INEGI (2000). Indicadores de desarrollo sustentable en México [en línea]. México: Instituto Nacional de Ecología/Instituto Nacional de Estadística, Geografía e Informática. Disponible en: http://www2.ine.gob.mx/publicaciones/ download/311.pdf [2008, 3 de julio].

Ivanova, A., et al. (2010). Alternative Tourism: a Pathway for Sustainability in the Cabo Pulmo National Park, Baja California Sur, Mexico. Indicators of Sustainability \& swot Analysis. IV Conferencia Inter-nacional en Turismo Sostenible. New Forest: Wessex Institute of Technology-UKA.

Lares Molina, O. y M. Á. López Flores (2004). "Metodología de diagnóstico para el desarrollo sustentable”. Revista del Centro de Investigación, 6 (22), juliodiciembre de 2004, 27-38. México: Universidad La Salle.

Lutz, E. (ed.) (1993). Toward Improved Accounting for the Environment. An UNSTATWorld Bank Symposium. Washington: The World Bank.

Martínez Alier, J. (1999). Introducción a la economía ecológica. Madrid: Rubes.

Max-Neef, M. (1991). Human Scale Development. Conception, Application and Further Reflections. Nueva York: The Apex Press.

Mondragón Pérez, A. R. (2002). “¿Qué son los indicadores?” Revista de In-formación

y Análisis, 19, 52-58. México: INEGI. 
Nieto, L. (2000-2001). “¿Cómo sabemos si tenemos avances hacia el desarrollo sostenible?” [en línea]. Disponible en: http://ambiental.uaslp.mx/docs/ LMNC-AP000799.pdf [2010, 23 de julio].

OCDE (1998). Towards Sustainable Development. Environmental Indicators. París: Organización para la Cooperación y el Desarrollo Económicos. (2003). OECD Environmental Indicators. Development, Measure-ment and Use [en línea]. Disponible en: http://www.oecd.org/dataoecd/7/47/24993546. pdf [2010, 21 de julio].

OMT (1997). Desarrollo turístico sostenible. Guía para planificadores locales. Madrid: Organización Mundial del Turismo.

PNUD (2008). Human Development Report 2007/2008. Nueva York: Programa de las Naciones Unidas para el Desarrollo.

Ponce Talancón, H. (2006). "La matriz FODA: una alternativa para reali-zar diagnósticos y determinar estrategias de intervención en las organizaciones productivas y sociales”. Contribuciones a la Economía, septiembre de 2006 [en línea]. Universidad de Málaga. Disponible en: http://www.eumed.net/ ce/2006/hpt-FODA.htm

Prescott-Allen, R. (1997). Consumption Patterns, Ecosystem Stress and Human Development. Washington, D. C.: Island Press.

Quiroga Martínez, R. (2001). Indicadores de sostenibilidad ambiental y de desarrollo sostenible: Estado del arte y perspectivas. Santiago de Chile: Comisión Económica para América Latina y el Caribe (CEPAL).

- (2007). Indicadores ambientales y de desarrollo sostenible: avances $y$ perspectivas para América Latina y el Caribe. Santiago de Chile: CEPAL.

Rotondo, E. y G. Vela (2004). Indicadores de género. Lineamientos concep-tuales y metodológicos para su formulación y utilización por los proyectos fida de América Latina y el Caribe. Lima: PREVAL/Progénero.

Sánchez, I. (2010). Indicadores de sustentabilidad para el manejo de la pesca rivereña; caso de San Evaristo y Bahía de La Paz. Tesis doctoral. La Paz: UABCS.

Schmidt-Bleek, F. (1994). Wieviel Umwelt braucht der Mensch?: Faktor 10 - das Maß für ökologisches Wirtschaften. Berlín: Verlag Birkhäuser. 
Sectur (2008). Modelo Tipo de Indicadores de Sustentabilidad. Desarrollo de un Modelo de Indicadores de Sustentabilidad para el Turismo [en línea]. México: Secretaría de Turismo. Disponible en: http://www.sectur.gob. mx/work/sites/sectur/resources/LocalContent/13329/3/Modelo_sistema_ indicadores.pdf [2008, 3 de julio].

Sejenovich, H. (1996). Hacia otro desarrollo, una perspectiva ambiental. Montevideo: Nordan.

Semarnat (2010). Los indicadores ambientales. Marco conceptual. Sistema Nacional de Indicadores Ambientales [en línea]. México: Secretaría de Medio Ambiente y Recursos Naturales. Disponible en: http://www.semarnat. gob.mx/informacionambiental/snia/documents/marco_conceptual.html [2010, 21 de julio].

Spangenberg, J. H. (1999). Critérios Integrados para a Elaboracao do conceito de sustentabilidade. Río de Janeiro: FASE.

Spangenberg, J. H. y F. Schmidt-Bleek (1997). "How Do We Probe the Physical Boundaries for a Sustainable Society?”, en L. Rydén (ed.). Foundations of Sustainable Development. Ethics, Law, Culture and the Physical Limits. Uppsala: Uppsala University, 37-44.

UICN (2001). Resource Kit For Sustentabilily Assessment. Gland: Unión Inter-nacional para la Conservación de la Naturaleza.

UNEP-WCMC (United Nations Environment Programme-World Conservation Monitoring Centre) (2000). Global Biodiversity: Earth's Living Re-sources in the 21st Century. Cambridge: World Conservation Press.

Vila, L. E., C. D. Dávila Quintana y J. G. Mora (2010). “Competencias para la innovación en las universidades de América Latina: un análisis empírico”. Revista Iberoamericana de Educación Superior, RIES, 1 (1), 5-23. México: Instituto de Investigaciones sobre la Universidad y la Educación-Universidad Nacional Autónoma de México/Universia.

Wackernagel, M. y W. Rees (1996). Our Ecological Footprint: Reducing Human Impact on the Earth. Gabriola Island: New Society Publishers.

Wautiez, F. y B. Reyes (2007). Indicadores locales para la sostenibilidad. La Habana: Instituto de Ecología Política/Acuario. 
Winograd, M., J. Eade Moldan y Billhartz (1997). Environmental and Sustainable Indicators for Latin America and the Caribbean: The Use for Geographical Information Systems, Sustainability Indicators.

WWF (1998-2008). Informes de un planeta vivo. Fondo Mundial para la Naturaleza.

— (2010). Metodología para la evaluación y priorización rápidas del manejo de áreas protegidas [en línea]. Disponible en: http://assets.panda.org/ downloads/finalrappamspanishsmall.pdf [2010, $1^{0}$ de octubre].

Xiaohua, S. (2007). "Call for Return to Green Accounting”. China Daily, 19 de abril de 2007,3 .

YCELP/CIESIN (2005). Environmental Sustainability Index. Davós: Yale Center for Environmental Law and Policy of Yale University/Center for International Earth Science Information Network of Columbia University. 